\title{
Electrolysis-reducing electrodes for electrokinetic devices
}

Per Erlandsson and Nathaniel D Robinson

\section{Linköping University Post Print}

N.B.: When citing this work, cite the original article.

This is the authors' version of the publication:

Per Erlandsson and Nathaniel D Robinson, Electrolysis-reducing electrodes for electrokinetic devices, 2011, ELECTROPHORESIS, (32), 6-7, 784-790.

http://dx.doi.org/10.1002/elps.201000617

Copyright: Wiley-VCH Verlag Berlin

http://www.wiley-vch.de/publish/en/

Postprint available at: Linköping University Electronic Press

http://urn.kb.se/resolve?urn=urn:nbn:se:liu:diva-67312 
DOI:

\title{
Electrolysis-reducing Electrodes for Electrokinetic Devices
}

By Per G. Erlandsson and Nathaniel D. Robinson*

[*] Dr. N. D. Robinson, P. G. Erlandsson

Transport and Separations Group, Dept. of Phys. Chem. and Bio., Linköping University, 58183 Linköping, (Sweden)

E-mail: natro@ifm.liu.se

\begin{abstract}
Abbreviations: CP, conjugated polymer; EC, electrochemical; EO, electroosmotic; EOP, electroosmotic pump; HCE, high concentration electrolyte; LCE, low concentration electrolyte; LOC, lab-on-a-chip; PEDOT, poly(3,4-ethylenedioxythiophene); PSS, poly(styrenesulfonate);
\end{abstract}

Keywords:

Conducting Polymers / Microfluidics / Electroosmosis / pH / gas evolution

Total number of words: 3883 
Submitted to Electrophoresis

\begin{abstract}
Direct current electrokinetic systems generally require Faradaic reactions to occur at a pair of electrodes to maintain an electric field in an electrolyte connecting them. The vast majority of such systems, e.g. electrophoretic separations (capillary electrophoresis) or electro-osmotic pumps, employ electrolysis of the solvent in these reactions. In many cases, the electrolytic products, such as $\mathrm{H}^{+}$and $\mathrm{OH}^{-}$in the case of water, can negatively influence the chemical or biological species being transported or separated, and gaseous products such as $\mathrm{O}_{2}$ and $\mathrm{H}_{2}$ can break the electrochemical circuit in microfluidic devices. This article presents an electroosmotic (EO) pump that employs the oxidation/reduction of the conjugated polymer poly(3,4-ethylenedioxythiophene) (PEDOT), rather than electrolysis of a solvent, to drive flow in a capillary. Devices made with PEDOT electrodes are compared to devices made with Pt electrodes in terms of flow and local $\mathrm{pH}$ change at the electrodes. Furthermore, we demonstrate that flow is driven for applied potentials under $2 \mathrm{~V}$, and the electrodes are stable for potentials of at least $100 \mathrm{~V}$.

Electrochemically active electrodes like those presented here minimize the disadvantage of integrated EO pumps in, e.g. lab-on-a-chip applications, and may open new possibilities, especially for battery-powered disposable point-of-care devices.
\end{abstract}




\section{Introduction}

Electrokinetic separations and electroosmotic pumps are the heart of many chemistry-related technologies, ranging from laboratory separations, e.g. capillary electrophoresis (CE), to microfluidic lab-on-a-chip (LOC) systems. The latter are predicted to significantly increase efficiency in the healthcare industry by replacing complex procedures involving large-scale chemistry and biology laboratory equipment with automated hand-held diagnostic tools. This, in turn, can help bring sophisticated diagnostic techniques to areas with limited laboratory facilities. The ease-of-manufacture and small size of electroosmotic pumps (EOPs) are obvious advantages for their use in LOC systems, however EOPs usually produce $\mathrm{H}_{2}$ and $\mathrm{O}_{2}$, $\mathrm{H}_{2} \mathrm{O}_{2}$ and/or $\mathrm{H}^{+}$and $\mathrm{OH}^{-}$at the electrodes [1-7]. All of these reactions are undesirable, as gases can quickly break the connection between the aqueous sample and an electrode, and $\mathrm{H}^{+}$, $\mathrm{OH}^{-}$and $\mathrm{H}_{2} \mathrm{O}_{2}$ can disturb sensitive materials (e.g. proteins) being studied. Still, it is beneficial to integrate the electrodes intimately with the fluidic system as the plug-like electroosmotic flow (EOF) associated with EOPs only exists between the electrodes. By taking advantage of the electrochemical (EC) functionality of materials such as conjugated polymers (CPs) used in organic electronics, we have created EOPs that nearly eliminate the oxidation/reduction of solvent, without electroplating e.g. Ag from one electrode to another, and hence the production of EC byproducts or release of materials that could interfere with biological and chemical samples. Using a blend of oxidized and reduced CP at each electrode means that electrochemistry, and hence fluid transport, begins at small applied potentials, as opposed to $\sim 2 \mathrm{~V}$ required for the case of Pt electrodes, making low-voltage battery-operated LOC devices easier to construct. It should also be noted that metal electrodes such as Pt, which often are considered to be inert, can undergo chemical changes and even dissolve when used as electrodes to drive EOF [8]. The polymers used here have the additional advantage of being solution processable, that is to say that they can be cast from solution using e.g. inkjet 
printing [9]. This combination makes these integrated pumps ideal for LOC applications, and also useful in a wide range of laboratory-scale systems.

Organic electronics is currently a very active research area in chemistry and materials science. Films of semiconducting polymers and molecules are already used in transistors and light-emitting diodes in electronic products available on the market. These devices almost exclusively employ the polymers' ability to transport electrons and holes, just as devices made of inorganic semiconductors such as $\mathrm{Si}$ or GaAs do. In other applications such as electrochromic windows and displays [10], light-emitting EC cells [11], and EC transistors [12], the EC interplay between CPs, ions and electrons is employed [13]. Although these EC devices were some of the first to be created in the lab, they are further from broad commercialization than their ion-free counterparts. However, it is the EC devices that expand the use of organic electronics towards "new" areas such as chemistry and microbiology [14], as hard materials are less hospitable for $e . g$. nerve cells than soft polymers [15]. The polymer system used here, poly(3,4-ethylenedioxythiophene) (PEDOT) blended with poly(styrenesulfonate) (PEDOT:PSS) [16], is commercially available and can be readily coated onto substrates or printed using e.g. inkjet, screen, or other technologies [9].

Previously, switching CPs like PEDOT between the oxidized and reduced states has been utilized to change the polymer's conductivity [12], optical absorbance [17, 18] and wettability $[19,20]$. Here, however, the change of redox state is used to allow an EC reaction to occur without generating mobile byproducts.

In short, EOF is the result of an electrokinetic force on the charged ions adjacent to an oppositely-charged surface [6]. The force is generated by an electric field applied along the length of the channel through which flow is desired. If the channel is small enough, e.g. $\sim 100$ $\mu \mathrm{m}$ or smaller in diameter, then the viscosity of the liquid is typically sufficient to cause the entire content of the capillary to move at the same velocity. The electric field that drives EOF is traditionally maintained by electrolytic reactions at two metal electrodes The reactions

$$
\text { Wiley- } \stackrel{4}{\mathrm{VCH}}
$$


change the $\mathrm{pH}$ both near the electrodes and in the system [6,7] and can generate gas bubbles that threaten to interrupt the contact between the fluid and electrode [1-3]. The side-reaction problem can be mitigated in laboratory-scale devices by using very large $($ e.g. $1 \mathrm{~L})$ liquid reservoirs around the electrodes or otherwise ensuring that the solution in proximity to the pump electrodes does not come near the sample [21, 22], or by adding chemical buffers to the sample [23]. The former strategy is difficult to employ in a miniaturized device as it requires a large footprint. The latter works in some cases, but adding new chemical components to an already complex biological sample comes with the risk of affecting the behavior of the material to be tested. Furthermore, the buffer is depleted near the electrodes. As a result, fullyintegrated EOPs based on the electrolysis of water are often unacceptable in LOC devices handling sensitive samples.

Several strategies have been employed to minimize the electrochemical reactions at EO pump electrodes [1-3]. For example, ion exchange membranes [4] have been designed to limit the impact of electrode reactions, however, these lowered pumping efficiency without removing the undesired reactions. Brask et al. present an AC EO pump utilizing valves to reduce the impact of reactions by alternating flow direction [24], but the advantage of pluglike flow is lost when using EO as a hydrostatic pressure source. Guzman et al. describe a low potential electrokinetic system with $\mathrm{Ag} / \mathrm{AgCl}$ electrodes which can induce reactions at voltages lower than necessary for electrolysis [25], however, the risk of releasing silver ions limits its practical use in microfluidics with e.g. proteins.

The device described in this article takes advantage of the ability of many semiconducting polymers like PEDOT to undergo EC changes, and uses this property to solve significant challenges in using EOPs and CE, particularly in miniaturized systems. The potential for using organic electronic materials as electrodes in chemical and biological systems has been demonstrated in two different types of electrophoretic devices [14, 26, 27]. In other applications for microfluidics, organic electronic materials have been used for 
electrochemically-controlled wettability $[19,28,29]$. Here, we demonstrate a metal-free EOP by using electrodes made of PEDOT:PSS to induce EOF through a glass capillary. When designed so that the PEDOT:PSS electrodes are never fully electrochemically switched, they are shown to have only a small impact on solution $\mathrm{pH}$ while driving flow in the capillary. Furthermore, repeated cycling of the device shows that oxidative degeneration [30, 31] (socalled electrochemical over-oxidation) of the PEDOT can be avoided even when potentials of over $100 \mathrm{~V}$ are employed.

\section{Materials and methods}

\subsection{Electrode Fabrication}

Electrodes were produced by drop-casting $100 \mu 1$ PEDOT:PSS (1.3 wt \% dispersion in $\mathrm{H}_{2} \mathrm{O}$, conductive grade, Sigma-Aldrich) containing 5\% diethylene glycol (Sigma-Aldrich) to cover a 20x7 $\mathrm{mm}^{2}$ area on "overhead transparency" film (3M, PP2500, polyester based). The diethylene glycol acts as a secondary dopant to increase the conductivity of the electrodes [32]. The transparency film $(60 \times 20 \mathrm{~mm})$ was roughened with sandpaper (P240, average particle diameter $58 \mu \mathrm{m}$ ) and bent at the middle after heating, creating self-standing L-shaped electrode substrates. After drop-casting, the PEDOT:PSS mixture was dried at room temperature, and stripes of silver conductive paint (Electrolube) were added to the top edge of each PEDOT:PSS electrode, as shown in Figure 1, to facilitate electronic contact to the instrumentation. Please note that Figure 1 is not to scale; for example the PEDOT:PSS films and capillary appear disproportionally thick. Since it is difficult to use a 4-point probe on soft polymer films like the one used here, the sheet resistance was measured by creating several square electrodes of varying size and measuring the resistance between silver electrodes applied to the full length of two opposite sides with a digital multimeter (Fluke 23). The 
PEDOT:PSS films produced showed a sheet resistance of roughly $20 \Omega /$ sq. The Pt electrodes used in the wells were $25 \mathrm{~cm}$ long, $0.2 \mathrm{~mm}$ diameter wires (EP1330, Sigma-Aldrich).

\subsection{Fluid Displacement Measurements}

The pumps consisted of two wells (40x30x12 mm) filled with aqueous salt solution, each containing a PEDOT:PSS electrode, connected with an untreated fused silica capillary, 10 mm long with a $25 \mu \mathrm{m}$ inner diameter. Fluid movement was verified with a displacement setup with wells containing differing electrolyte concentrations, 5 and $20 \mathrm{mM}$ of both $\mathrm{Na}_{2} \mathrm{HPO}_{4}$ (Sigma-Aldrich) ( $\mathrm{pH}$ 9.4) and $\mathrm{NaCl}(\mathrm{pH}$ 5.7), $10 \mathrm{~mL}$ per well. The potential was applied and the current through the system measured by a Keithley 2636A Source-Meter.

\section{3 pH Measurements}

The comparison between PEDOT and Pt electrodes was performed with $20 \mathrm{mM} \mathrm{NaCl}$ as electrolyte, $10 \mathrm{~mL}$ per well, adding $10 \mathrm{mM} \mathrm{NaOH}$ and $\mathrm{HCl}$ solution until the electrolyte was stable at around $\mathrm{pH}$ 5.1. The electrolyte is more sensitive to the generation of $\mathrm{H}^{+}$and $\mathrm{OH}^{-}$at this $\mathrm{pH}$ than at e.g. $\mathrm{pH} 7$ due to the buffering effects of carbonic acid from atmospheric $\mathrm{CO}_{2}$. To make any $\mathrm{pH}$ changes due to electrolysis apparent, no buffer was added to the electrolyte. The pump used a $10 \mathrm{~mm}$ long, $50 \mu \mathrm{m}$ i.d. fused silica capillary and was driven at a constant current of $4 \mu \mathrm{A}$. The driving current was periodically paused by opening the electric circuit so that the $\mathrm{pH}$ of the electrolyte in each electrode well could be measured. Then, the current was resumed until the next $\mathrm{pH}$ measurement. During these measurements, the $\mathrm{pH}$ of a control sample, a well containing the same electrolyte without any electrode, was also measured to quantify any $\mathrm{pH}$ changes from e.g. $\mathrm{CO} 2$ interactions. All electrolyte $\mathrm{pH}$ measurements were performed with a VWR SympHony SB70P pH-meter.

\subsection{Cyclic Voltammetry Measurements}

All CV measurements were performed in a three-electrode electrochemical cell in $20 \mathrm{mM}$ $\mathrm{NaCl}$ with a platinum counter electrode and an $\mathrm{Ag} / \mathrm{AgCl}(3 \mathrm{M} \mathrm{NaCl})$ reference electrode connected to a $\mu$ Autolab type II potentiostat/galvanostat (Metrohm Autolab). The

$$
\text { Wiley-ver }
$$


PEDOT:PSS electrode used for the voltammogram in Figure 1 was approximately $1 \mathrm{~cm}$ by 2 $\mathrm{cm}$ and $5 \mu \mathrm{m}$ thick. The corresponding Pt working electrode had a surface area of $2 \mathrm{~mm}^{2}$. The sweep rate during the $\mathrm{CV}$ measurements was $25 \mathrm{mV} / \mathrm{s}$. Autolab Software (GPES) was used to capture and analyze the CV data.

\subsection{Potential drop across the capillary}

The potential, during operation, across a $10 \mathrm{~mm}$ long, $50 \mu \mathrm{m}$ i.d. capillary in the pumping setup filled with $20 \mathrm{mM} \mathrm{NaCl}$ was measured using two $\mathrm{Ag} / \mathrm{AgCl}$ reference electrodes (BASinc RE-5B) connected to one channel of a Keithley 2636 source-meter.

\subsection{Electrode lifetime measurements}

Pumps with PEDOT electrodes, $10 \mathrm{~mm}$ long, $25 \mu \mathrm{m}$ i.d. capillaries and $20 \mathrm{mM} \mathrm{NaCl}$ electrolyte were run with an applied potential of $100 \mathrm{~V}$. The polarity was reversed every two hours, reversing the pumping direction. After cycling the pump for 37 days, the PEDOT electrodes' functionality was verified again by cyclic voltammetry and by testing their ability to pump without modifying the electrolyte $\mathrm{pH}$ as previously described. The charge transferred per cycle during a 37-day lifetime measurement and a subsequent $\mathrm{pH}$ measurement with the same electrodes is presented in the supplementary information.

\section{Results and Discussion}

When in contact with an electrolyte, switching PEDOT between the p-doped and undoped state (or vice versa) requires ions $\left(\mathrm{M}^{+}\right)$to leave (or enter) the polymer to maintain local electroneutrality, as shown in Figure 1. The reduction half-reaction for PEDOT is shown in equation 1. This reaction is reversible, i.e. the PEDOT can be doped via the associated oxidation. Through these half reactions, the PEDOT effectively acts as an electron-to-ion transducer [33], allowing an EC reaction to take place at each electrode without causing electrolysis.

$\mathrm{PEDOT}^{+} \mathrm{PSS}^{-}+\mathrm{M}^{+} \rightarrow \mathrm{PEDOT}^{0}+\mathrm{M}^{+} \mathrm{PSS}^{-}$

$$
\text { Wiley-vcH }
$$


The half-reaction shown in (1) occurs well within the EC stability window of water [16], as verified by cyclic voltammetry and shown in Figure 2. Since it starts as a mixture of doped and undoped PEDOT, each polymer electrode (solid curve) performs electrochemistry even at small applied potentials, allowing significant charge to be transferred where electrolysis is limited (e.g. between 0.3 and $-0.3 \mathrm{~V}$ vs. $\mathrm{Ag} / \mathrm{AgCl}$ ). Besides reducing the generation of byproducts, this enables pump operation at low potentials, e.g. those supplied by batteries in portable devices. After the PEDOT is exhausted (oxidized or reduced), in the CV sweep, e.g. over $0.5 \mathrm{~V}$ or below $-0.5 \mathrm{~V}$ vs. $\mathrm{Ag} / \mathrm{AgCl}$ in Figure 2, electrolysis of water becomes significant. We wish to note that the entire volume of each PEDOT electrode is available for oxidation/reduction because PEDOT:PSS forms a hydrogel in water that readily transports ions [34]. In the pump, this means that a significant amount of electrolyte can be pumped before the device needs to be turned off or the pumping direction reversed. Furthermore, before the electrodes are exhausted (fully oxidized or reduced), electrolysis is insignificant compared to the electrochemistry of PEDOT even when much larger external potentials are applied. Although this device was demonstrated with PEDOT, similar devices can almost certainly be produced with electrodes made from a wide variety of electrochemically active conducting polymers and metal oxides. The most important factors are that the EC change in the electrodes' redox state occurs within the EC stability window of the solvent and that the material remains within the electrode rather than being released into the electrolyte.

The pumps were manufactured by placing a silica capillary between two wells in a rectangular 8-well polystyrene dish. The relatively large reservoirs compared to the volume of the capillary allowed easy measurement of $\mathrm{pH}$, freer electrode design and lower impact from evaporation. While the size of electrodes and wells helped to reliably measure the difference between electrode materials, the size also made the system too large to be considered microfluidic. However, the observed impact of electrolysis should be equally or even more 
important when scaling down the system. A PEDOT:PSS electrode was placed in each well, the wells were filled with electrolyte, and a potential applied between the electrodes.

A simple equivalent circuit and schematic of the electrostatic potential within the pump together with experimental measurements shown and described in the supplementary information illustrate that the majority of the applied potential drops across the capillary. However, the potential drop at the platinum electrodes increases with increasing applied potential while the drop remains low at PEDOT electrodes, see supplementary information. With small capillary diameters and sufficient undoped (doped) electrode material in the anode (cathode), this allows large potentials to be applied without risk of over-oxidizing the PEDOT, an irreversible reaction which permanently reduces the electrode's capacity [30, 31].

Filling the two wells with salt solutions of differing concentration allowed us to easily estimate the flow rate through the capillary without the use of dyes, particles, or other additives, as the current transported through the capillary for a given electric field is proportional to the ion concentration of the electrolyte within the capillary [35], see the supplementary information for a detailed description.

The resistance of the capillary, calculated from the measured current assuming that the capillary is the only significant resistance, is plotted as a function of time for a sequence of potentials applied between the PEDOT:PSS electrodes as shown in Figure 3. In this device, the capillary was $10 \mathrm{~mm}$ in length, with an inner diameter of $25 \mu \mathrm{m}$. The high and low concentration electrolytes (HCE and LCE, respectively) were $20 \mathrm{mM}$ and $5 \mathrm{mM}$ solutions of $\mathrm{Na}_{2} \mathrm{HPO}_{4}$, respectively. The applied potential (solid line in Figure 3a) was varied to drive the flow (points in Figure 3a) in both directions and at several flow rates.

For the experimental sequence shown in Figure 3, the capillary was initially filled with the LCE. Upon changing the applied potential from $+5 \mathrm{~V}$ to $-5 \mathrm{~V}$ at approximately 300 seconds, the HCE was introduced into the capillary. As the HCE displaced the LCE, the resistance within the capillary (inversely proportional to the average concentration within the 
capillary) decreased (nearly) linearly until the capillary was filled with the HCE, at which point it reached a minimum. Notice that this does not indicate that the pump stopped transporting electrolyte, but simply that the ionic resistance across the capillary no longer changed. The flow velocity, shown as points in Figure 3a, was calculated by multiplying the time derivative of the resistance with the capillary length divided by the difference between the minimum and maximum in the resistance trace. Notice that differentiating the recorded current results in a relatively noisy signal. We believe the real flow rate to be much steadier than indicated. Only the values of the flow velocity where the calculation can be performed accurately (i.e. while a concentration front was propagating through the capillary) are shown in Figure 3a. Reversing the potential, for example from $-5 \mathrm{~V}$ to $+5 \mathrm{~V}$ at approximately $800 \mathrm{~s}$ in Figure 3, caused the flow to reverse as anticipated. The resistance increased (nearly) linearly as the LCE progressively filled the capillary. The density of negative charge on the capillary wall depends on the $\mathrm{pH}$ of the solution, changing from $20 \mathrm{mM} \mathrm{NaHPO} 4$ (pH 9.4) to $\mathrm{NaCl}(\mathrm{pH}$ 5.7) nearly cuts the EOF in half. The flow velocity for $20 \mathrm{mM} \mathrm{NaCl}$ (pH 5.7) for potentials between 2 and $80 \mathrm{~V}$ is shown in Figure 4. These single-capillary pumps are not optimal for generating a large pressure; a multi-channel design would achieve higher pressure and flow rates with the same cross sectional area [36].

Since the primary reason for using electrochemically-active electrodes is to reduce the effects of the electrolysis of water, we measured the change in electrolyte $\mathrm{pH}$ in EOPs with PEDOT electrodes and compared them to pumps made with Pt electrodes. Pumps, like those shown in Figure 1, were operated continuously in one direction at a constant current of $4 \mu \mathrm{A}$, resulting in an applied potential around $80 \mathrm{~V}$. The average $\mathrm{pH}$ of measurements from four separate PEDOT devices as a function of transferred charge is shown in Figure 5. The error bars on the PEDOT data represent two standard deviations in the measurements. The reference value was measured in a well without electrodes to detect any $\mathrm{pH}$ changes due to temperature and atmospheric effects. Using the polymer electrodes, the $\mathrm{pH}$ remained stable 
within one tenth of a $\mathrm{pH}$ unit until $10 \mathrm{mC}$ was transferred. In comparison, the same change of $\mathrm{pH}$ occurred during the first $2 \mathrm{mC}$ with Pt electrodes, and when $10 \mathrm{mC}$ was transferred, the $\mathrm{pH}$ difference between the wells exceeded 0.8 . Note that both the $\mathrm{pH}$ change and associated error bars are larger for the cathode than for the anode, which is to be expected since the $\mathrm{pH}$ is the negative logarithm of proton concentration, making the measurement more sensitive closer to $\mathrm{pH} 7$.

Although they can operate for a relatively long time, the electrodes used in this EOP are finite and can be "exhausted" (oxidized at the anode or reduced at the cathode) so the pump cannot operate in one direction indefinitely. When one or both electrodes are fully exhausted, electrolysis of water at the polymer/electrolyte interface takes over and the pump continues to operate in a manner similar to an EOP based on metal electrodes. This is evident in the increase (decrease) in $\mathrm{pH}$ at the cathode (anode) visible for the PEDOT data in Figure 5 for transferred charge $>10 \mathrm{mC}$. It is therefore important to design and operate the pump such that the electrodes are never exhausted, in this case up to about $10 \mathrm{mC}$.

As with the materials in secondary (rechargeable) batteries, repeatedly charging and discharging the PEDOT electrodes in the EOP could cause undesired side-reactions within the polymer that limit the electrodes' lifetime, particularly at elevated potentials [30, 31]. The significance of this effect was investigated by reversing a $100 \mathrm{~V}$ potential applied between PEDOT:PSS electrodes in an EOP every 2 hours, resulting in a charge transfer close to $10 \mathrm{mC}$ during each cycle. The charge transferred during each cycle under a 37-day measurement shows no deterioration, and the same electrodes were subsequently used in the $\mathrm{pH}$ measurement presented in the supplementary information, verifying that they maintained their functionality.

Although the pump described here has macroscopic vessels and electrodes, the target area of application is in the micro and even nano scale. However, there is no reason to expect difficult challenges in scaling such a device. Techniques such as soft lithography and 
photolithography are frequently used to etch capillaries and vessels in glass, and CP electrodes can be inkjet-printed [9] into such wells with a resolution of a few microns. When reducing the electrode size, the charge that can be transferred before electrolysis decreases significantly. Our results indicate approximately $10 \mathrm{mC}$ of charge per mg (dry weight) of PEDOT:PSS in the electrodes is available for pumping before electrolysis becomes important. Other studies using PEDOT:PSS show that the electrodes are highly stable in various other solutions/buffers, apart from those containing oxidizing agents which can cause overoxidation of the PEDOT [37, 38].

\section{Concluding remarks}

Using conducting and electrochemically-switchable materials as electrodes, we have demonstrated an EOP that drives aqueous samples without significant electrolysis, i.e. without generating gas or changing the solution's $\mathrm{pH}$. Furthermore, the pump begins operating at potentials smaller than the electrochemical stability window of water, making it a strong candidate for use in portable battery-driven devices. In this example, we employed the CP PEDOT; however, other conducting materials, e.g. CPs or metal oxides, function as electronto-ion transducers in a similar manner. The key criteria are that the electrode material be conductive and that it can be oxidized/reduced within the EC stability window of the electrolyte used. The concept should also work for electrodes in, e.g., capillary or gel electrophoresis systems. It is our hope that the biocompatible nature of these materials, coupled with the ease with which solution-based manufacturing can be performed, will facilitate and accelerate the development and use of LOC devices in a wide variety of applications. 


\section{References}

[1] Arnold, D. W., Paul, P. H., Schoeniger, J. S., US Patent 6,287,440 2001

[2] Khamizov, R. K., Kumakhov, M. A., Bastrukina, N. S., Voronov, A. A., Nikitina, S. V., US Patent 20100346672009

[3] Liu, S., Lu, J., US Patent 200201665922002

[4] Brask, A., Kutter, J., Bruus, H., Lab Chip 2005, 5, 730-738.

[5] Wang, P., Chen, Z., Chang, H., Sens Actuators B 2006, 113, 500-509.

[6] Persat, A., Suss, M., Santiago, J., Lab Chip 2009, 9, 2454-2469.

[7] Minerick, A. R., Ostafin, A. E., Chang, H.-C., Electrophoresis 2002, 23, 2165-2173.

[8] Gencoglu, A., Minerick, A., Lab Chip 2009, 9, 1866-1873.

[9] Mannerbro, R., Ranlof, M., Robinson, N., Forchheimer, R., Synthetic Met 2008, 158, 556560 .

[10] Nielsen, C. B., Angerhofer, A., Abboud, K. A., Reynolds, J. R., J Am Chem Soc 2008, 130, 9734-9746.

[11] Pei, Q. B., Yu, G., Zhang, C., Yang, Y., Heeger, A. J., Science 1995, 269, 1086-1088.

[12] Thackeray, J. W., White, H. S., Wrighton, M. S., J Phys Chem-Us 1985, 89, 5133-5140.

[13] Leger, J. M., Adv Mater 2008, 20, 837-841.

[14] Isaksson, J., Kjall, P., Nilsson, D., Robinson, N. D., et al., Nat Mater 2007, 6, 673-679.

[15] Tee, S. Y., Bausch, A. R., Janmey, P. A., Curr Biol 2009, 19, R745-R748.

[16] Groenendaal, B. L., Jonas, F., Freitag, D., Pielartzik, H., Reynolds, J. R., Adv Mater 2000, $12,481-494$.

[17] Mortimer, R. J., Dyer, A. L., Reynolds, J. R., Displays 2006, 27, 2-18.

[18] Schwendeman, I., Hickman, R., Sonmez, G., Schottland, P., et al., Chem Mater 2002, 14, 3118-3122.

[19] Robinson, L., Hentzell, A., Robinson, N. D., Isaksson, J., Berggren, M., Lab Chip 2006, 6, 1277-1278.

[20] Wang, X., Ederth, T., Inganas, O., Langmuir 2006, 22, 9287-9294.

[21] Chuan-Hua, C., Santiago, J. G., J Microelectromech S 2002, 11, 672-683.

[22] Culbertson, C. T., Ramsey, R. S., Ramsey, J. M., Anal Chem 2000, 72, 2285-2291.

[23] Crabtree, H. J., Cheong, E. C. S., Tilroe, D. A., Backhouse, C. J., Anal Chem 2001, 73, 4079-4086.

[24] Brask, A., Snakenborg, D., Kutter, J. P., Bruus, H., Lab Chip 2006, 6, 280-288.

[25] Guzman, K. A. D., Karnik, R. N., Newman, J. S., Majumdar, A., J Microelectromech S 2006, 15, 237-245.

[26] Henderson, R. D., Guijt, R. M., Haddad, P. R., Hilder, E. F., et al., Lab Chip 2010, 10, $1869-1872$.

[27] Isaksson, J., Nilsson, D., Kjall, P., Robinson, N. D., et al., Org Electron 2008, 9, 303-309. [28] Isaksson, J., Tengstedt, C., Fahlman, M., Robinson, N., Berggren, M., Adv Mater 2004, $16,316-+$.

[29] Causley, J., Stitzel, S., Brady, S., Diamond, D., Wallace, G., Synthetic Met 2005, 151, 60-64.

[30] Tehrani, P., Kanciurzewska, A., Crispin, X., Robinson, N. D., et al., Solid State Ionics 2007, 177, 3521-3527. 
[31] Tehrani, P., Isaksson, J., Mammo, W., Andersson, M. R., et al., Thin Solid Films 2006, $515,2485-2492$.

[32] Crispin, X., Jakobsson, F., Crispin, A., Grim, P., et al., Chem Mater 2006, 18, 4354-4360. [33] Bobacka, J., Anal Chem 1999, 71, 4932-4937.

[34] Li, G. C., Pickup, P. G., Phys Chem Chem Phys 2000, 2, 1255-1260.

[35] Mela, P., Tas, N. R., Berenschot, E. J. W., van Nieuwkasteele, J., van den Berg, A., Electrophoresis 2004, 25, 3687-3693.

[36] Edwards, J. M., Hamblin, M. N., Fuentes, H. V., Peeni, B. A., et al., Biomicrofluidics 2007, 1, 014101.

[37] Nikolou, M., Malliaras, G. G., Chem Rec 2008, 8, 13-22.

[38] Thaning, E. M., Asplund, M. L., Nyberg, T. A., Inganas, O. W., von Holst, H., J Biomed Mater Res B Appl Biomater 2010, 93, 407-415. 
Submitted to Electrophoresis

\begin{abstract}
Acknowledgements
The authors thank the Swedish Science Council (Vetenskapsrådet) for funding of this project.

Supporting Information is available online from Wiley InterScience or from the author.
\end{abstract}




\section{Conflict of interest statement}

The authors have submitted a patent application regarding the device described in this article and its use (Swedish patent application number: 1050168-2). 
Submitted to Electrophoresis

Figure captions

Figure 1. A schematic of the electroosmotic pump tested in the laboratory, including the electrochemical half-reactions occurring at the anode and cathode and the transport of electrons and cations (e.g. $\left.\mathrm{Na}^{+}\right)$within the device.

Figure 2. Cyclic voltammogram of Pt (dotted line) and PEDOT:PSS (solid line) electrodes in $20 \mathrm{mM} \mathrm{NaCl}_{(\mathrm{aq})}$. Arrows indicate the oxidation and reduction peaks associated with the PEDOT.

Figure 3. Measurement of flow velocity. a) Applied potential (solid line) and calculated flow velocity (points) through the EOP as a function of time. b) Measured current (dashed line) and calculated resistance across the capillary (solid line).

Figure 4. Flow velocity versus potential for $20 \mathrm{mM} \mathrm{NaCl}(\mathrm{pH} 5.7)$ through a $25 \mu \mathrm{m}$ i.d. 10 mm long fused silica capillary and PEDOT electrodes. Velocities were measured in both directions (driven by positive and negative potentials) in the symmetric device, but shown here only as positive values for simplicity. Data shown are the average of three measurements, error bars are within symbol size.

Figure 5. Measured electrolyte $\mathrm{pH}$ at the anode (square) and cathode (circle) versus accumulated charge transferred for PEDOT and Pt electrodes. The average value of four measurements is shown with error bars equal to twice the standard deviation 
Figure 1. A schematic of the electroosmotic pump tested in the laboratory, including the electrochemical half-reactions occurring at the anode and cathode and the transport of electrons and cations (e.g. $\mathrm{Na}+$ ) within the device.

\section{$254 \times 190 \mathrm{~mm}(600 \times 600 \mathrm{DPI})$}


Figure 2. Cyclic voltammogram of Pt (dotted line) and PEDOT:PSS (solid line) electrodes in 20mM $\mathrm{NaCl}(\mathrm{aq})$. Arrows indicate the oxidation and reduction peaks associated with the PEDOT. $288 \times 201 \mathrm{~mm}(600 \times 600 \mathrm{DPI})$ 
1

2

3

4

5

6

7

8

9

10

11

12

13

14

15

16

17

18

19

20

21

22

23

24

25

26

27

28

29

30

31

32

33

34

35

36

37

38

39

40

41

42

43

44

45

46

47

48

49

50

51

52

53

54

55

56

57

58

59

60
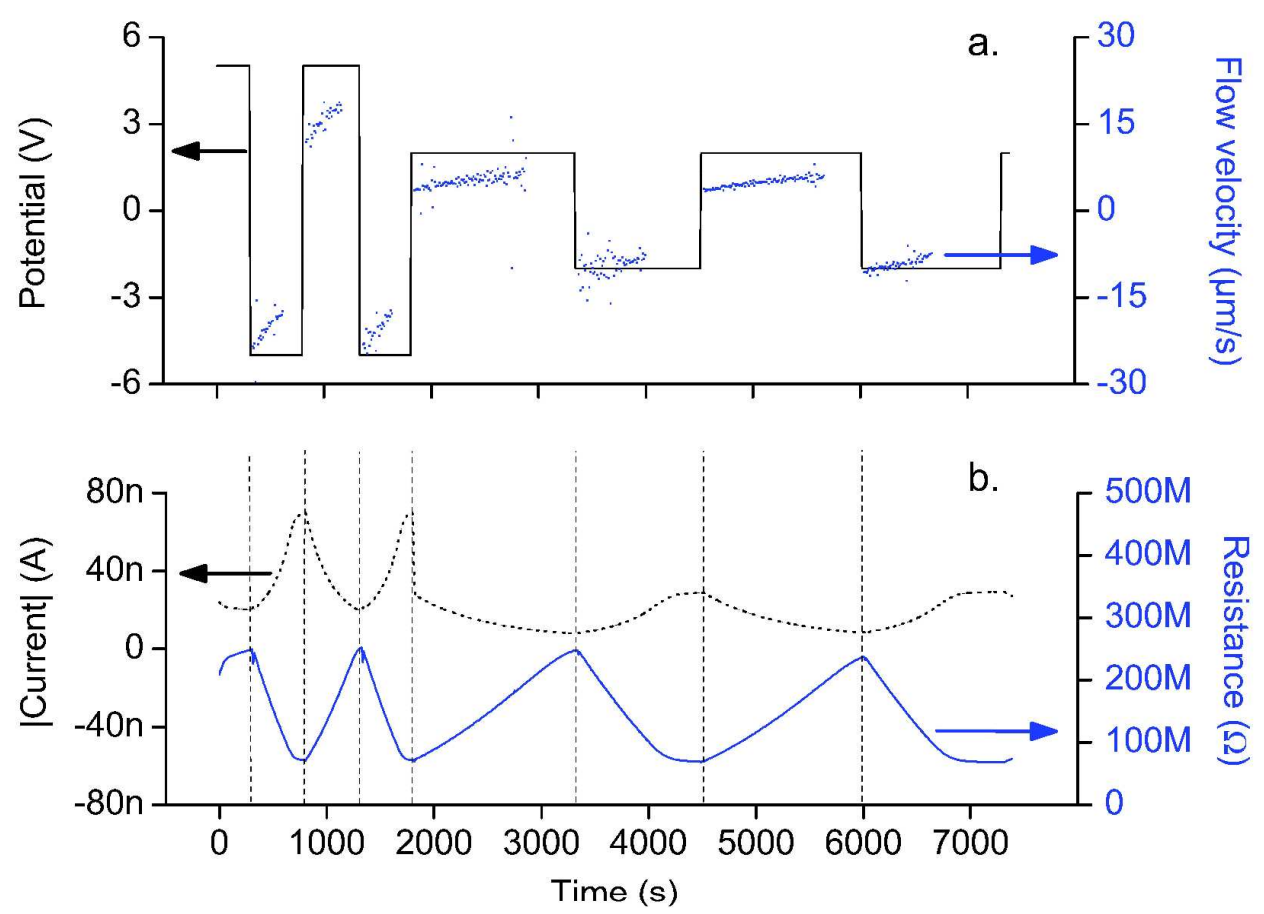

Figure 3. Measurement of flow velocity. a) Applied potential (solid line) and calculated flow velocity (points) through the EOP as a function of time. b) Measured current (dashed line) and calculated resistance across the capillary (solid line). $288 \times 201 \mathrm{~mm}(600 \times 600 \mathrm{DPI})$ 


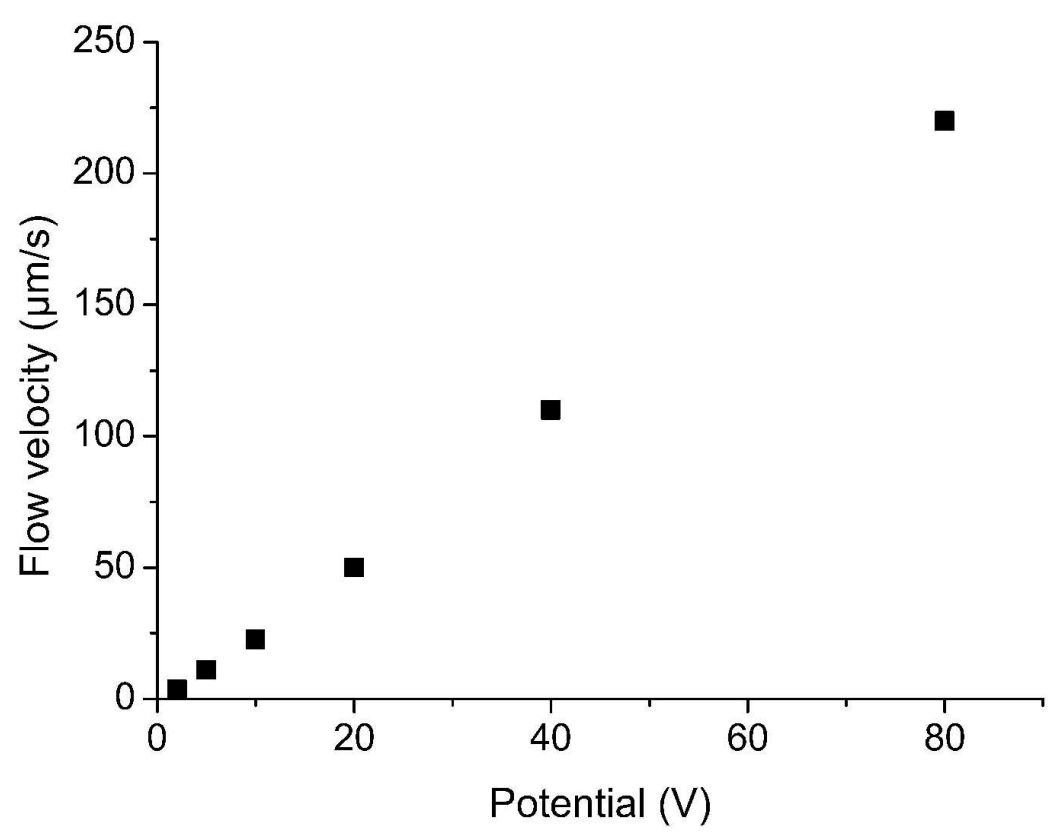

Figure 4. Flow velocity versus potential for $20 \mathrm{mM} \mathrm{NaCl}(\mathrm{pH} \mathrm{5.7)} \mathrm{through} \mathrm{a} 25 \mu \mathrm{m}$ i.d. $10 \mathrm{~mm}$ long fused silica capillary and PEDOT electrodes. Velocities were measured in both directions (driven by positive and negative potentials) in the symmetric device, but shown here only as positive values for simplicity. Data shown are the average of three measurements, error bars are within symbol size.

$288 \times 201 \mathrm{~mm}(600 \times 600 \mathrm{DPI})$ 


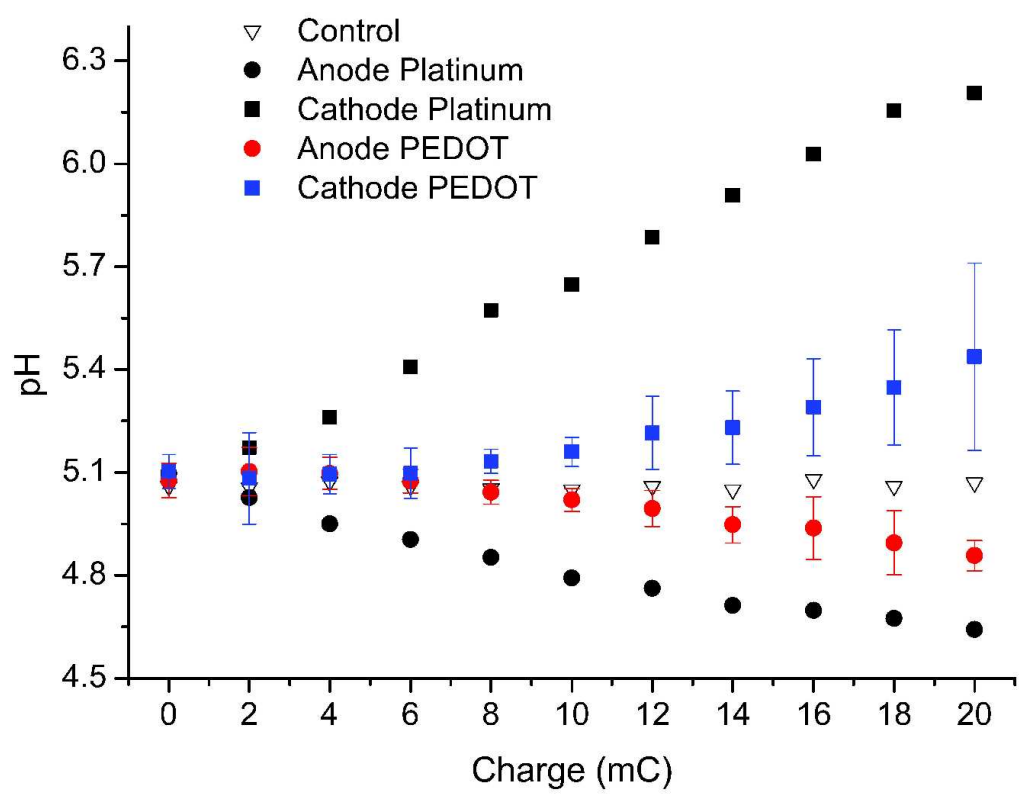

Figure 5. Measured electrolyte $\mathrm{pH}$ at the anode (square) and cathode (circle) versus accumulated charge transferred for PEDOT and Pt electrodes. The average value of four measurements is shown with error bars equal to twice the standard deviation

$$
288 \times 201 \mathrm{~mm}(600 \times 600 \text { DPI })
$$

